\title{
Influence Mechanism of Secondary Gel Technique on Bi-2212 Superconducting Phase: Gel Models Simulation and Verification
}

\author{
Huazhe Yang ${ }^{2 *}$, M. Babar Shahzad ${ }^{1,3}$, Xiaoming $\mathrm{Yu}^{1,3}$, Yang Qi $^{1^{*}}$ \\ ${ }^{1}$ Institute of Materials Physics and Chemistry, School of Sciences, Northeastern University, \\ Shenyang, 110819, China \\ ${ }^{2}$ Department of Physics, China Medical University, Shenyang 110122, China \\ ${ }^{3}$ Institute of Metal Research, Chinese Academy of Sciences, Shenyang 110016, China
}

\begin{abstract}
Study of gel structures as precursors for $\mathrm{Bi}_{2} \mathrm{Sr}_{2} \mathrm{Ca}_{1} \mathrm{Cu}_{2} \mathrm{O}_{\mathrm{x}}(\mathrm{Bi}-2212)$ superconductors can provide better understanding of the role of gel process in the synthesis of superconducting phase. However, it is still challenging to investigate the amorphous gel system through conventional methods. Here, based on our former experiments, we present a computer simulation method to establish the geometric structural models of ethylenediaminetetracetic acid (EDTA)-metal cation complexes, conventional gel and home-made secondary gel. Local orbital density functional method, $\mathrm{Dmol}^{3}$ approach was applied to optimize the structures and compare the thermodynamical stabilities of different systems. In addition, Bi-2212 samples with different sintering time were prepared via both conventional and secondary gel technique to verify the calculation results. Results demonstrated that the secondary gel system possesses higher phase stability compared to the conventional gel owing to its higher bonding ability of EDTA-metal cation complexes. Hence, component homogeneity of complexes can be enhanced and pure Bi-2212 phase can be synthesized with shorter sintering time.
\end{abstract}

Key words: secondary gel; Bi-2212; thermodynamic calculation; component homogeneity

\section{Introduction}

$\mathrm{Bi}_{2} \mathrm{Sr}_{2} \mathrm{Ca}_{\mathrm{x}-1} \mathrm{Cu}_{\mathrm{x}} \mathrm{O}_{\mathrm{y}}$ (BSCCO) superconductors have attracted significant attention due to its higher critical temperature $\left(\mathrm{T}_{\mathrm{c}}\right)$ and critical current density, etc., which are more auspicious relative to other superconductors, e.g. $\mathrm{MgB}_{2}$ and $\mathrm{YBa}_{2} \mathrm{Cu}_{3} \mathrm{O}_{7-\mathrm{x}}$ [1-10]. These features make BSCCO systems promising as electric power transmission lines, fault current limiters, transformers and electromagnets. Also, epitaxial BSCCO films have been considered as a good candidate to obtain Josephson superconducting devices [11, 12].

Up to date, significant contributions have been made to develop techniques for the synthesis

\footnotetext{
* Corresponding author. Tel.:+86 2423256666

E-mail address: hzyang@mail.cmu.edu.cn (HuazheYang); qiyang@imp.neu.edu.cn (Yang Qi)
} 
of BSCCO phase [13-17]. The sol-gel technique is considered as one of the most effective way to reduce the impurities and save processing time by providing intimate mixing of metal cations on the atomic scale [18-22]. However, synthesis of pure $\mathrm{Bi}_{2} \mathrm{Sr}_{2} \mathrm{Ca}_{1} \mathrm{Cu}_{2} \mathrm{O}_{\mathrm{x}}$ (Bi-2212) or $\mathrm{Bi}_{2} \mathrm{Sr}_{2} \mathrm{Ca}_{2} \mathrm{Cu}_{3} \mathrm{O}_{\mathrm{x}}(\mathrm{Bi}-2223)$ phases is still seen to be a difficult task owing to the complicated ingredients of its precursor. As a result, the intermediate grinding of precursor powder or calcinations for a long time seem to be unavoidable to obtain pure BSCCO powder, which is time and energy consuming.

Recently, based on ethylenediaminetetracetic acid (EDTA)-metal cation complexes, we have developed a novel technique named "secondary gel technique" to synthesize $\mathrm{Bi}_{2} \mathrm{Sr}_{2} \mathrm{Ca}_{1} \mathrm{Cu}_{2} \mathrm{O}_{\mathrm{x}}$ (Bi-2212) superconducting phase [23-25]. Compared with conventional gel technique, the phase purity of Bi-2212 powders was enhanced clearly via secondary gel technique as verified through the X-ray diffraction (XRD) analyses. Furthermore, influence of different gel processes on structure of the precursor gel, homogeneity of ingredients and superconducting behavior of Bi-2212 samples was investigated. We presumed that the secondary gel technique could intensify the gel network during the transformation from the conventional gel to secondary gel resulting in enhanced precursor homogeneity and BSCCO phase purity [24]. However, there is insufficient evidence of such presumption, especially, the gel network is amorphous and has a low stability, and it is difficult to investigate the gelatin process in detail by using existing experimental methods such as Fourier Transform Infrared Spectroscopy (FTIR) and XRD analysis.

Compared with the experimental studies, computational studies have advantages in dealing with an unstable and complicated system [26, 27]. Therefore, it is feasible to investigate the thermodynamic properties of gel system via computational modeling and calculation in order to clarify the mechanism of secondary gel technique during the synthesis of Bi-2212 phase. To the best of our knowledge there are virtually no published theoretical studies investigating the role of gelation process in BSSCO phase using gel models.

In this study, based on our former investigation, models of EDTA-metal cation complexes and different types of gel were established. The local orbital density functional (DF) method, $\mathrm{DMol}^{3}$ approach, was used to investigate the influence mechanism of secondary gel technique on Bi-2212 phase by comparing the thermodynamic stability of the conventional gel and secondary gel systems. In addition, different sintering times of the precursors derived from the two gel techniques were used to provide the supporting information for stability of different gel systems. Overall, this investigation not only provides a theoretical explanation of our former experimental results but also brings novel insights into the mechanism of gelation process during the formation of BSCCO phase.

\section{Calculation and experimental}

\subsection{Model establishment and calculation}

The visualized Materials studio software (Accelrys Company) was applied to establish the models of the monomer complexes, water molecular and the precursor gel. The Dmol ${ }^{3}$ module based on DF method was used to optimize the structure and to calculate the energies of the systems.

According to an earlier investigation [28], EDTA can complex with a metal ion with six coordination bonds (Fig.1). Models of the geometric structures of the EDTA-Bi, EDTA-Sr, 
EDTA-Ca and EDTA-Cu monomer complexes were respectively established manually according to the above structure by adding and binding different atoms using Materials Studio software. Afterwards, a water molecular model was also established manually according to its molecular formula, i.e., one oxygen atom and two hydrogen atoms. In order to diminish the structural inaccuracies brought by the manual modelling, structure of the monomer complexes and water molecules were optimized by using "Geometry optimization" process in Dmol ${ }^{3}$ module, where the "Quality" for the "Geometric optimization" module was chosen as "medium" and the "SCF tolerance" and "Max. SCF cycles" were automatically set to $1.0 \mathrm{e}^{-5}$ and 50 respectively by the software interface (see the Supporting Information S1). According to our former experimental assumption, structural models of conventional gel and secondary gel were established by binding different monomer complexes with hydrogen bond or water molecules respectively in proper molar ratio, i.e. EDTA-Bi, EDTA-Sr, EDTA-Ca and EDTA-Cu in a ratio of 2:2:1:2, respectively, which was in accordance with the molar ratio of different metal cations of Bi-2212. The simplified complexes models were established according to the basic unit $\left(\mathrm{Bi}_{2} \mathrm{O}_{3}\right.$-SrO-CuO-CaO-CuO-SrO- $\left.\mathrm{Bi}_{2} \mathrm{O}_{3}\right)$ of $\mathrm{Bi}-2212$ crystal and the bonding mode of hydrogen bonds between adjacent complexes (molar ratio of water molecular and complexes is 2:1). As a result, the simplified number, i.e. 7 complexes and 14 water molecules were adopted. In addition, since only a basic unit of gel model was established, a circular shape of the combination of monomer complexes and water molecular was set for both conventional and secondary gel in order to decrease the boundary effect (the asymmetric influence on the boundary complexes in the selected basic unit part). After the "Geometry optimization" process, the parameters such as the energy convergence, gradient convergence and displacement convergence were also adjusted automatically to optimize the calculation. Finally, the binding energy of different gel systems was calculated to compare the stability of different systems.

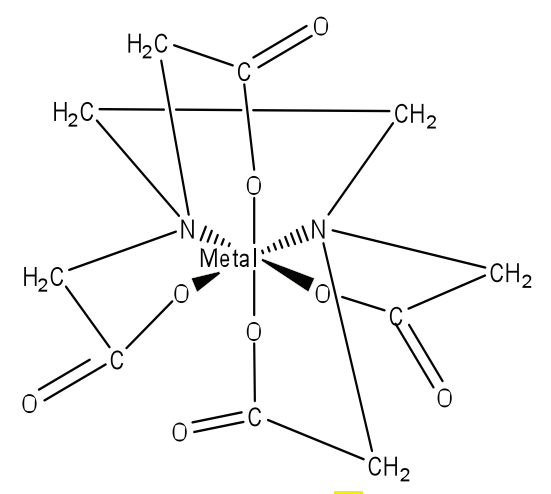

Fig. 1 Structure of EDTA-metal ion complex

\subsection{Verification experiment}

According to the model and calculation, verification experiment was performed through sol-gel method. Metal nitrates were used as reactants and EDTA as chelating agent to obtain a blue mixed solution. The conventional precursor sol and gel was formed by heating the solution at $65^{\circ} \mathrm{C}$. The secondary gel was formed at a higher temperature $\left(150^{\circ} \mathrm{C}\right)$ after the occurrence of the precursor gel was occurred. After further heating and self-combustion, the dark-brown precursor powder was obtained. Finally, Bi-2212 powders derived from different gels were obtained at $850^{\circ} \mathrm{C}$ for different sintering time, i.e., $10 \mathrm{~h}, 5 \mathrm{~h}, 3 \mathrm{~h}$ and $1 \mathrm{~h}$ respectively. The detailed experimental procedure is described in $[24,25]$. XRD $(\mathrm{Cu}-\mathrm{K} \alpha)$ and standard four-probe analyses were performed to 
investigate the crystallinity and temperature dependence of resistance (R-T) of samples.

\section{Results and discussion}

\subsection{Gel models and their stabilities}

Structural models of EDTA-metal cation monomer complexes are shown in Fig.S2 (Supporting Information). No remarkable structural changes in all EDTA-metal cation monomer complexes were detected before (Fig.S2(a)) and after (FigS2(b) ) the "Geometry optimization" process except for the bond angle and bond length. In addition, compared to the EDTA-Bi, EDTA-Sr and EDTA-Ca complexes, a minimal structural change was detected in EDTA-Cu. Total energies and convergences for different complexes after the optimization are listed in Table $\mathrm{S} 1$ (Supporting Information). In the table, $1(\mathrm{au})=627.51 \mathrm{kcal} / \mathrm{mol}$, and it is shown that EDTA-Bi complex possess lowest total energy of $-21181.98(\mathrm{au})$ (corresponds $-1.33 \times 10^{7} \mathrm{kcal} / \mathrm{mol}$ ). Similarly, there are also no obvious structural changes for water molecule model before (Fig.S3(a)) and after (Fig.S3(b)) the "Geometry optimization" process (Supporting Information). The total energy of water molecule after the structural optimization is -75.89 (au), i.e., $-4.77 \times 10^{4} \mathrm{kcal} / \mathrm{mol}$.

It is well known that degree of sol-gel transformation can influence the resulting superconducting phase and the component segregation in the gel can be magnified and lead to impurities in the samples. According to our previous experimental results [24], compared to conventional gel technique, secondary gel technique can increase Bi-2212 phase purity remarkably. Furthermore, according to the results of FTIR analysis, different states of water molecules exist in different gels, i.e., water of free state exits in conventional gel while water of binding state exits in secondary gel. It was presumed that the bonding state of water changed during the transformation from conventional gel to secondary gel, leading to change of association mode of hydrogen bonds to bind different EDTA-metal cation complexes. The proposed structural models of conventional gel and secondary gel are shown in Fig.2.

(a)

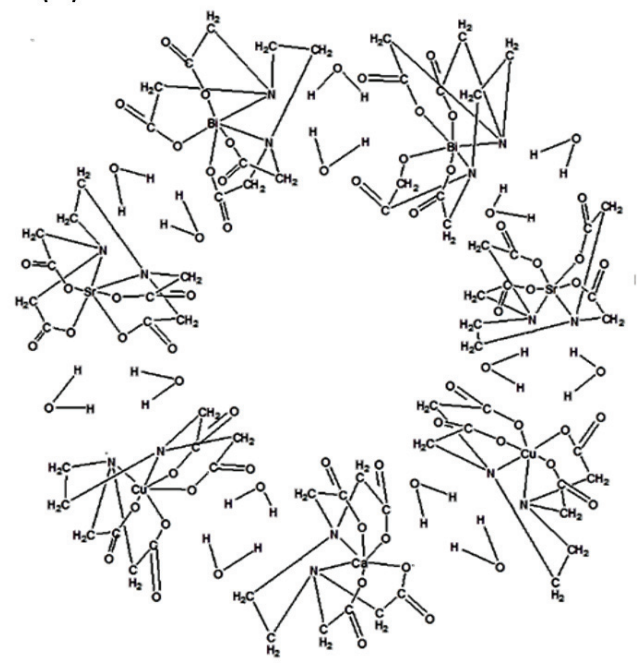

(b)

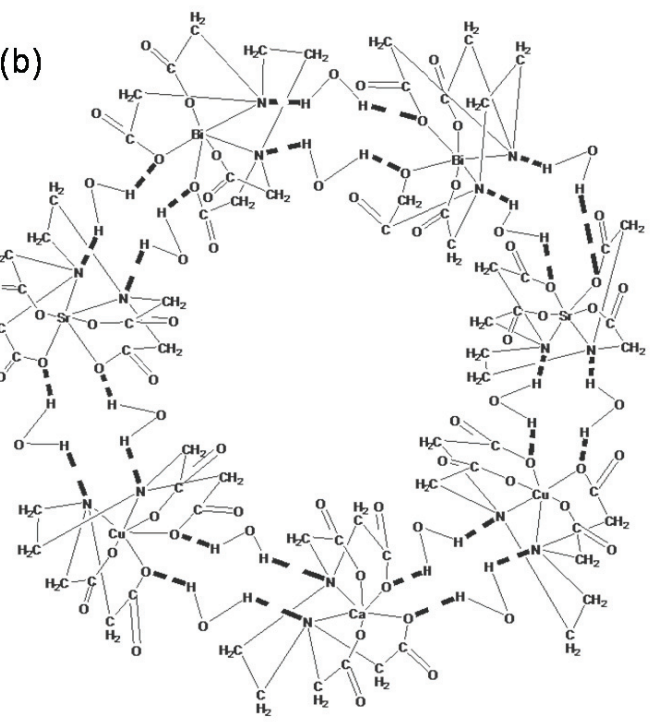

Fig.2 Proposed structural models of (a) conventional gel and (b) secondary gel

Fig.2 (a) and (b) is the structural models of conventional and secondary gel, respectively. In the conventional gel, the dominant bond to bind each EDTA-metal cation complexes is the 
hydrogen bond of free-state water, which is wrapped in the gel skeleton, and the weak bonding of free water can almost be neglected. It means that the skeleton of the conventional gel is mutable and sensitive to temperature, and ingredient segregation can take place immediately when heating the gel directly to the precursor powder. While in secondary gel, the free-state water is incorporated in the gel skeleton as bound water, and the bond between the complexes is replaced by an $-\mathrm{H}-\mathrm{O}-\mathrm{H}-$ bond during the transformation of gel to secondary gel. Therefore, the segregated component produced owing to the weak bonding ability of conventional gel can be reintegrated into the skeleton of the gel structure, which improves the chemical homogeneity of the gel. As a result, the Bi-2212 phase purity increases via secondary gel technique.

In order to provide further evidence for this presumed mechanism, the Material Studio software was used to investigate the structural and thermodynamic changes from conventional gel to secondary gel. In conventional gel, the bonding energy between different EDTA-metal cation complexes is weak since there is still a high amount of free water wrapped in the gel skeleton. Thus, the total energy of the conventional gel can be calculated from the following equation:

$$
E_{\text {total }}=\sum n_{i} \times E_{i}
$$

where $E_{\text {total }}$ is the total energy of the gel, $i$ is the category of components, and $n$ is the number of the specific component.

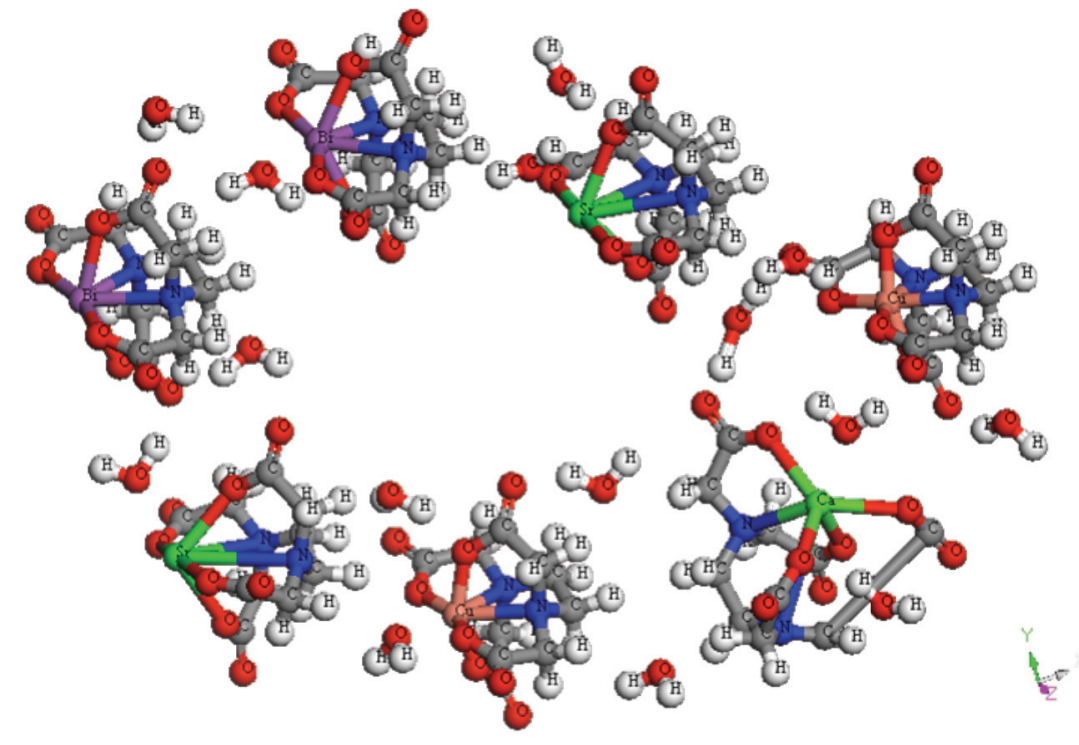

Fig.3 Structural models of secondary gel after structural optimization

The total energy of the conventional gel is the sum of energies for different complexes and water molecules, which is -59094.27 (au). In secondary gel, the water is incorporated into the gel skeleton as bound water and the distance between EDTA-metal cation complexes is short, therefore, the interaction between the complexes can no longer be neglected. The optimized structural model of secondary gel is shown in Fig.3. Compared to the models before optimization (Fig.S4, Supporting Information), both the positions and angels of the complexes and the water are changed. As well-known, structural changes of bond angle and bond length in monomer complexes and water can influence the partial charge distribution, which may further influence the stability of the whole gel system. The systematical investigation on influence of the structural changes on stability of systems is in progress. In addition, according to the calculation results, the 
total energy of secondary gel is -59167.76 (au) after the optimization, which is -73.49 (au) $\left(-4.61 \times 10^{4} \mathrm{kcal} / \mathrm{mol}\right)$ lower than that for conventional gel. The higher thermodynamic stability in secondary gel can account for the stronger complexes binding in the gel. As a result, the segregated component can decrease and the homogeneity of complexes in secondary gel can be improved. Results from the calculation are in good accordance with our previous experiment [23-25], which can clarify the mechanism of secondary gel technique.

\subsection{Influence of gel technique on Bi-2212 phase}

According to the results from the calculation as well as our former experiment, sintering time to synthesize Bi-2212 phase is speculated to decrease through secondary gel technique due to higher homogeneity of complexes in secondary gel. In order to further verify the speculation, both the conventional gel and secondary gel were heated at $850^{\circ} \mathrm{C}$ to synthesize Bi-2212 phase. Meanwhile, different sintering time was applied to investigate the influence of gel technique on the sintering time.

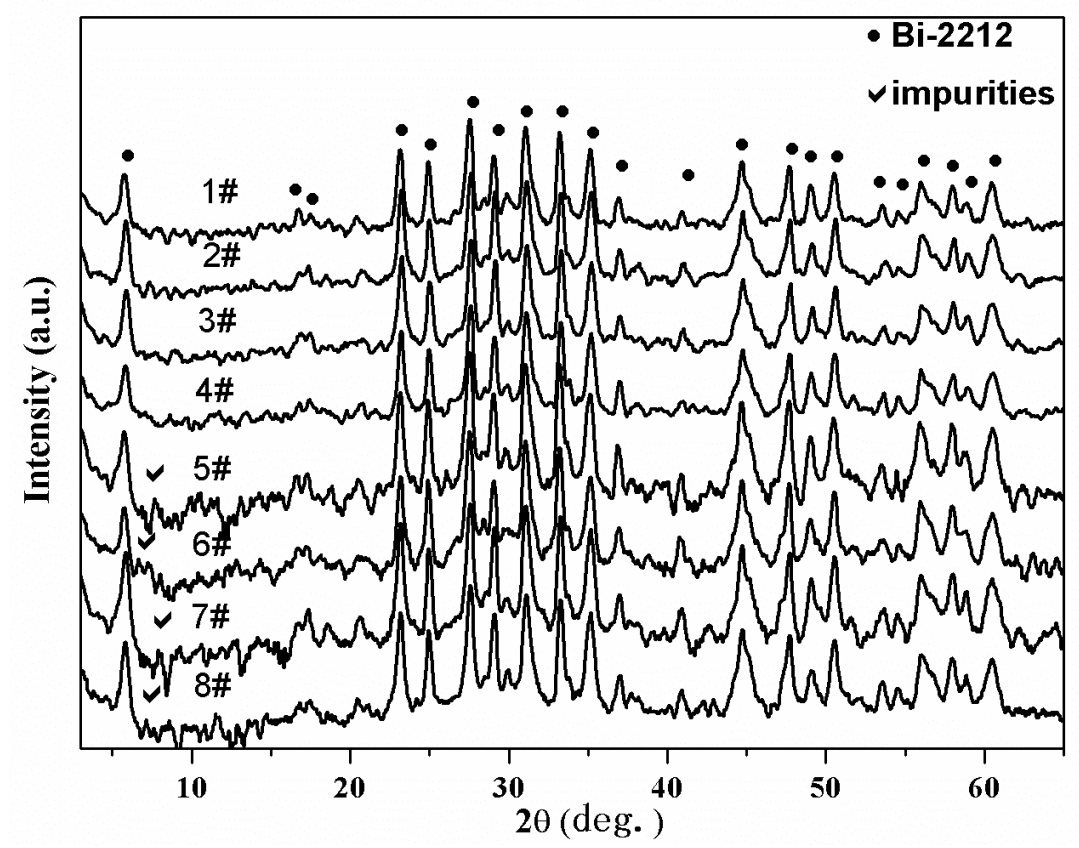

Fig.4 XRD patterns of Bi-2212 powder prepared by conventional (1\#, 3\#, 5\# and 7\#) and secondary gel technique (2\#, 4\#,6\# and 8\#). The sintering time for sample $1 \#-8 \#$ was $10 \mathrm{~h}, 10 \mathrm{~h}, 5 \mathrm{~h}$, $5 \mathrm{~h}, 3 \mathrm{~h}, 3 \mathrm{~h}, 1 \mathrm{~h}$ and $1 \mathrm{~h}$, respectively.

XRD patterns of Bi-2212 powders derived from precursor gel technique (1\#, 3\#, 5\# and 7\#) and secondary gel technique (2\#, 4\#,6\# and 8\#) with different sintering time are shown in Fig.4, where the sintering time for sample $1 \#-8 \#$ was $10 \mathrm{~h}, 10 \mathrm{~h}, 5 \mathrm{~h}, 5 \mathrm{~h}, 3 \mathrm{~h}, 3 \mathrm{~h}, 1 \mathrm{~h}$ and $1 \mathrm{~h}$, respectively. The peak intensity was normalized for all samples for convenience in comparison of Bi-2212 phase purity. Nearly pure Bi-2212 phase was found in samples $1 \#-4 \#$, while impurities such as Bi-2201 phase emerged in samples 5\#-8\#. Therefore, sintering time of less than $5 \mathrm{~h}$ is insufficient for metallic cations to diffuse and react to form pure Bi2212 phase, and the Bi2201 impurities can be formed for both of the gel techniques. In order to further compare the slight phase difference and crystallinity in two types of samples especially for samples $1 \#-4 \#$, the angular offset and full width at half maximum (FWHM) values of (002) peaks of samples were calculated as shown in 
table 1. According to the relationship between molar fraction of Bi2212 phase in the intergrowth phase and the (002) peak position of mixed BSCCO crystal [29, 30], the molar fractions of Bi2212 phase $(1-\mathrm{X})$ in the $(\mathrm{Bi} 2212)_{1-\mathrm{x}}(\mathrm{Bi} 2201)_{\mathrm{x}}$ intergrowth phases can be obtained by following formula:

$$
X=\left(\theta-\theta_{2212}\right) /\left(\theta_{2201}-\theta_{2212}\right)
$$

where $\theta$ is the diffraction angle of intergrowth phase, $\theta_{2212}$ and $\theta_{2201}$ is the diffraction angle of pure Bi2212 and Bi2201 phase from standard JCPDS data, respectively. Accordingly, the value of 1-X for sample $1 \#-8 \#$ is $93 \%, 86 \%, 86 \%, 90 \%, 93 \%, 96 \%, 89 \%$ and $90 \%$, respectively. Therefore, the samples derived from the secondary gel technique possess higher component (molar fractions) of Bi2212 phase in the intergrowth phases except for samples with sintering time of $10 \mathrm{~h}$. In addition, FWHM values of samples derived from secondary gel technique were also lower than that of conventional gel technique in most cases. Overall, secondary gel technique is more favorable to enhance Bi-2212 phase purity and crystallinity especially for samples with sintering time lower than $10 \mathrm{~h}$. Furthermore, the desirable sintering time for samples prepared by conventional gel technique is $10 \mathrm{~h}$ (sample1\#) regarding the phase purity. The decrease of sintering time led to the decrease in molar fractions of Bi2212 phase in the (Bi2212) $)_{1-\mathrm{x}}(\mathrm{Bi2201})_{\mathrm{x}}$ intergrowth phases (sample 3\#) along with the presence of impurities (samples 5\# and 7\#) . In contrast, for samples prepared by secondary gel technique (2\#, 4\#,6\# and 8\#), the requested sintering time is considered to be $5 \mathrm{~h}(4 \#)$. Alteration of sintering time will lead to the decrease in phase purity and crystallinity. Therefore, from view of phase purity and crystallinity, the requested sintering time for the formation of Bi-2212 phase is $10 \mathrm{~h}$ for conventional gel technique and $5 \mathrm{~h}$ for secondary gel technique. Thus, secondary gel technique can decrease the sintering time up to half for the formation of pure Bi-2212 phase with desirable crystallinity. The further investigation on the detail optimal sintering time for secondary gel technique is in progress.

Table 1 Parameters of (002) peak in sample 1\#-8\#

\begin{tabular}{ccc}
\hline Samples & Offset $/ \mathrm{O}$ & FWHM \\
\hline $1 \#$ & & \\
\hline $2 \#$ & 0.10 & 0.38 \\
$3 \#$ & 0.20 & 0.32 \\
$4 \#$ & 0.21 & 0.27 \\
$5 \#$ & 0.15 & 0.20 \\
$6 \#$ & 0.12 & 0.60 \\
$7 \#$ & 0.06 & 0.32 \\
$8 \#$ & 0.16 & 0.35 \\
\hline
\end{tabular}

Temperature dependence of resistance for samples 1\#-4\# are shown in Fig.5, where resistances of samples were normalized. Fig.5 (b) shows the enlarged view of marked area in Fig.5 (a). The $\mathrm{T}_{\mathrm{c} \text { (onset) }}$ of samples $1 \#-4 \#$ is $101 \mathrm{k}, 101 \mathrm{k}, 102 \mathrm{k}$ and $103 \mathrm{k}$, respectively, while $\mathrm{T}_{\mathrm{c} \text { (zero) }}$ of the four samples is $83 \mathrm{k}, 82 \mathrm{k}, 80 \mathrm{k}$ and $83 \mathrm{k}$, respectively. In addition, a distinct residual resistance at 77 
$\mathrm{K}$ can be observed in samples $3 \#$, which demonstrates that it is unsuitable to decrease the sintering time from $10 \mathrm{~h}$ to $5 \mathrm{~h}$ for conventional gel technique. In case of secondary gel technique, there is no distinct deterioration in $\mathrm{T}_{\mathrm{c}}$, which proves that the decrease of sintering time will not decrease superconductivity of samples. Consequently, secondary gel technique can decrease the sintering time from $10 \mathrm{~h}$ to $5 \mathrm{~h}$ for the formation of desired Bi-2212 phase, which is in accordance with the results obtained from XRD patterns.

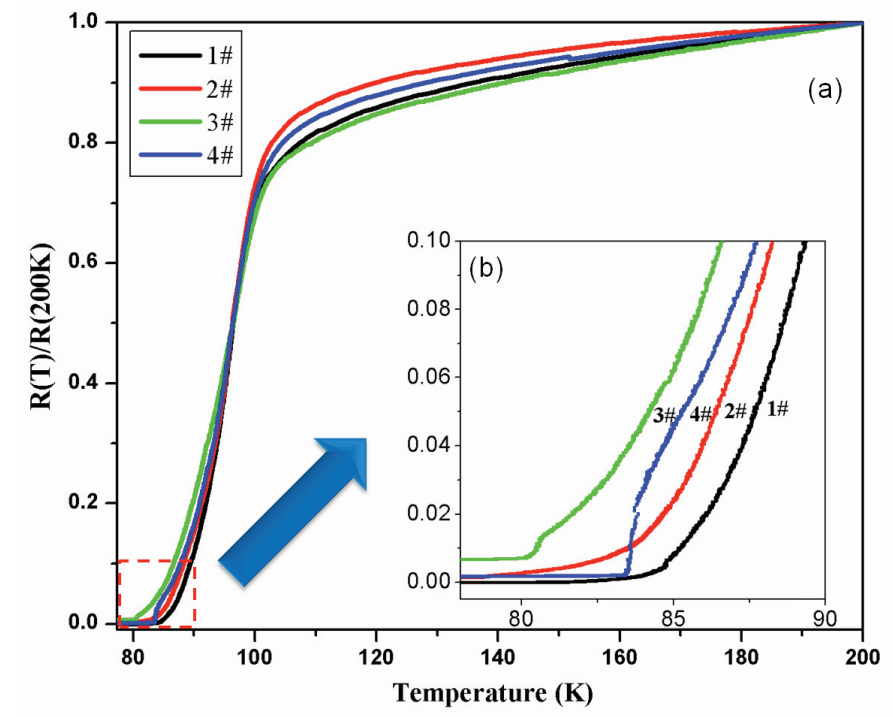

Fig. 5 Temperature dependence of resistance for samples $1 \#-4 \#$

According to the XRD and R-T results, it is verified that secondary gel has better chemical homogeneity of components due to the reintegration of segregated complexes. In this way, the energy barrier for the diffusion of metal ions to form Bi-2212 phase decrease and the sintering time to form $\mathrm{Bi}-2212$ phase can be reduced. Therefore, results of verification experiments are in accordance with the calculation results.

\section{Conclusions}

Mechanism of secondary gel technique to synthesize Bi-2212 phase was investigated and verified by both theoretical calculation and experimental results. It was proved that the model of secondary gel system, whose energy is with $4.61 \times 10^{5} \mathrm{kcal} / \mathrm{mol}$ lower than conventional gel system, is thermodynamically more stable than conventional gel. Accordingly, the initially segregated complexes caused by the weak bonding ability of conventional gel were reintegrated into the skeleton of secondary gel during the gel transformation. As a result, a better chemical homogeneity of EDTA-metal cation complexes can be obtained, and the sintering time for the formation of pure Bi-2212 phase can decrease from $10 \mathrm{~h}$ to $5 \mathrm{~h}$ via secondary gel technique.

\section{Acknowledgements}

This work was financially supported by the National Science Foundation of China (Grant No. 51172040; No. 81500897), China Scholarship Council (No. 201408210385), Foundation of the Education Department of Liaoning Province in China (No. L2013285) and Science and 
Technology Planning Project of Shenyang City (No. F11-262-9-16). The authors would like to acknowledge Professor Anmin Zheng from Wuhan Institute of Physics and Mathematics, Chinese Academy of Sciences, for offering the computing environment of $\mathrm{Dmol}^{3}$ modules.

\section{References}

[1] I. Chong, Z. Hiroi, M. Izumi, J. Shimoyama, Y. Nakayama, K. Kishio, T. Terashima, Y. Bando, M. Takano, Science, 1997, 276 (2):770-773

[2] S. Safran, H. Ozturk, M. Alp, E. Asikuzun, and O. Ozturk, Journal of Materials Science: Materials in electronics, 2015, 26: 2622-2628

[3] S. Watanabe, K. Hamanaka, T. Tachiki, T. Uchida, Physica C, 2009, 469:818-821

[4] E.A. Duarte, P.A. Quintero, M.W. Meisel, J.C. Nino, Physica C, 2013, 495: 109-113

[5] S.A. Halim, S.A. Khawaldeh, S.B. Mohamed, H. Azhan, Materials Chemistry and Physics, 1999, 61:251-259.

[6] B. Özçelik, O. Nane, A. Sotelo, and M. A. Madre, Ceramics Internatioanl, 2016, 42(2): 3418-3423

[7] A. Sotelo, B. Ozcelik, H. Amaveda, A. Bruned, and M. A. Madre, Ceramics Internatioanl, 2015, 41(10): 14276-14284

[8] R. M. S. Marhaba, M. R. R. Awad, and M. K. I. Hassan, Journal of Superconductivity and Novel, 2014, 27: 1131-1142

[9] D.M. Gokhfel'd, D.A. Balaev, S.V. Semenov, M.I. Petrov, Physics of the Solid State, 2015, 37(11):2145-2150

[10] H.Z. Yang, X.G. Sun, X.M. Yu, Y. Qi, Applied surface Science, 2012, 261:364-368

[11] I. Kakeya, T. Yamzaki, M. Kohri, T. Yamamoto, k. Kadowaki, Physica C, 2006, 437-438:118-121

[12] R. Kleiner, F. Steinmeyer, G. Kunkel, P. Müller, Physical Review Letters, 1992, 68: 2394 2397

[13] M. Abrecht, D. Ariosa, M. Onellion, G. Margaritondo, D. Pavuna, Journal of Applied Physics, 2002, 91:1187-1190

[14] S. Watanabe, K. Hamanaka, T. Tachiki, T. Uchida, Physica C, 2009, 469: 818 - 821

[15] Z. Mori, T. Doi, D. Kawabata, K. Ogata, K. Takahashi, A. Matsumoto, H. Kitaguchi, Y. Hakuraku, Physica C, 2008, 468:1060-1063

[16] B. Özçelik, H. Gündoğmuş, and D. Yazıcı, J. Mater. Sci. Mater. Electron., 2014, 25(6): 2456-2462

[17] O.V. Kharissova, E.M. Kopin, V.V. Maltsev, N.I. Leonyuk, L.M. Leon-Rossano, I.Y. Pinus, B.I. Kharisov, Critical Reviews in Solid State and Materials Sciences, 2014, 39:253-276

[18]H. S. W. Chang, S. R. Sheen, D. H. Chen, C. T. Chang, C. H. Kao, J. C. Huang, Y. C. Hsieh, Y. H. Hsieh and M. K. Wu, Mater. Lett, 1993, 16: 342-346.

[19]M. T. Ruiz, G. F. de la Fuente, A. Badía, J. Blasco, M.Castro,A. Sotelo,A. Larrea, F. Lera, C. Rillo, and R.Navarro, J. Mater. Res, 1993, 8: 1268-1276.

[20] R. Flükiger, A. Jeremie, B. Hensel, E. Seibt J. Q. Xu, and Y. Yamada, Adv. Cryo. Eng., 1992, 38: 1073

[21] V. Rouessac, J. Wang, J, Provost and G. Desgardin, J. Mat. Sci, 1996, 31: 3387-3390.

[22] Yang Kook Sun and Wha Young Lee, Physics C, 1993, 212:37-42.

[23] H.Z. Yang, X.M. Yu, Y. Ji, Y. Qi, Applied Surface Science, 2012, 258(11):4852-4856

[24] H.Z. Yang, W.S. Chen, Z.Z. Zhi, W. Wei, C.B. Zhang, Y. Qi, Material Science Forum, 2007, 
546-549: 1993-1996

[25] Y. Zhang, H.Z. Yang, M.L. Li, B.Z. Sun, Y. Qi, CrystEngComm,2010, 12:3046-3051

[26] C. Liu, H.Z. Yang, P. Wan, K.H Wang, L.L. Tan, K. Yang, Materials Science and Engineering: $C$, 2014, 35(1):1-7

[27] H.Z. Yang, C. Liu, P. Wan, L.L. Tan, K. Yang, APL Materials, 2013, 1(5):052104-1-052104-7

[28] F. H. Chen, H. S. Koo, T. Y.Tseng, J. Am. Ceram. Soc., 1992, 75(1): 96-102

[29] L.Ranno, D.Martinez-Garcia, J.Perriere, P.Barboux, Phys.Rev. B., 1993, 48(18):13945-13948

[30] H. Ota, K. Sakai, Z. Mori, R. Aoki, Appl. Phys. Lett., 1997, 70(11): 1471-1473 

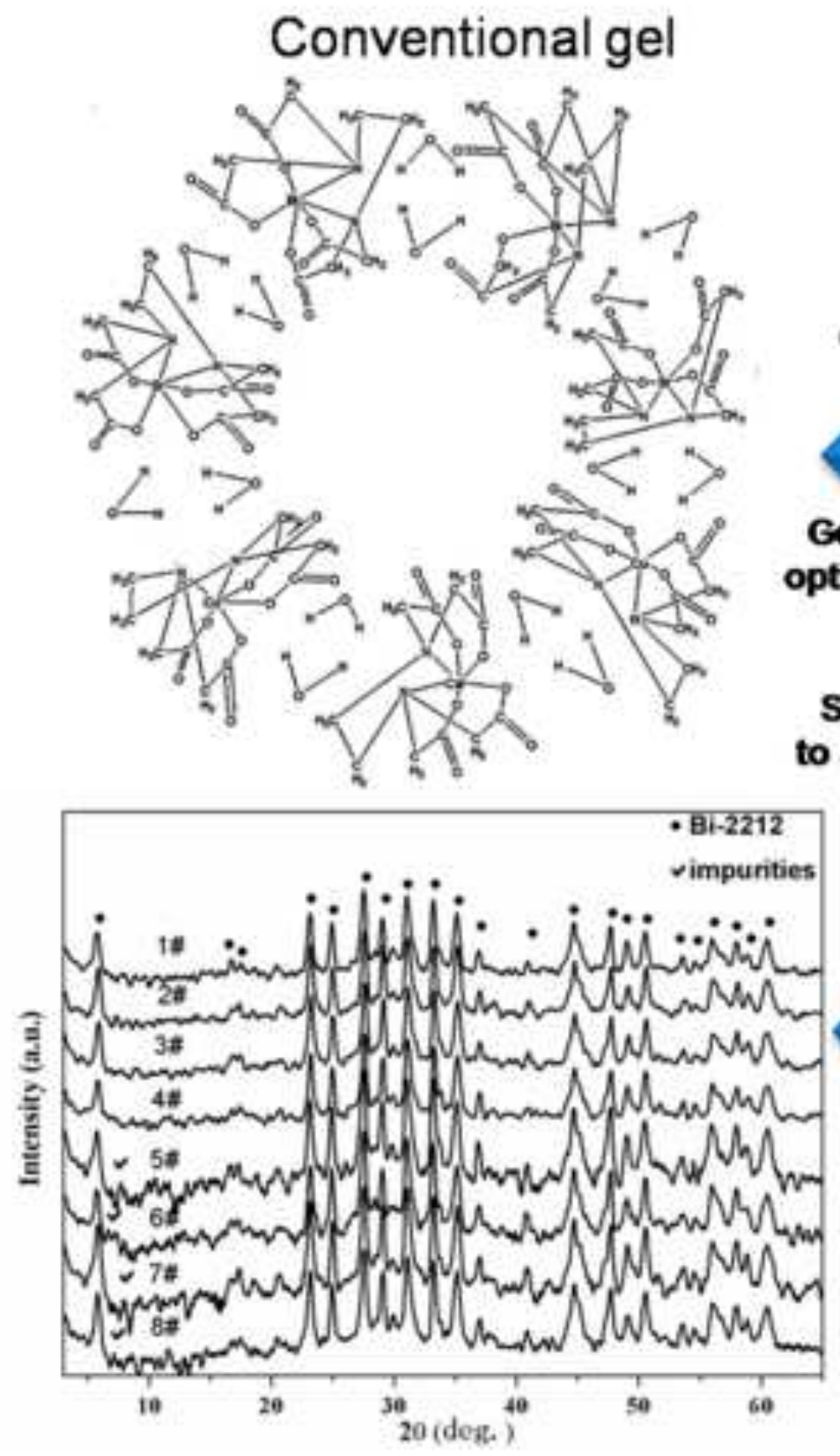

\section{Change of bonding between complexes}

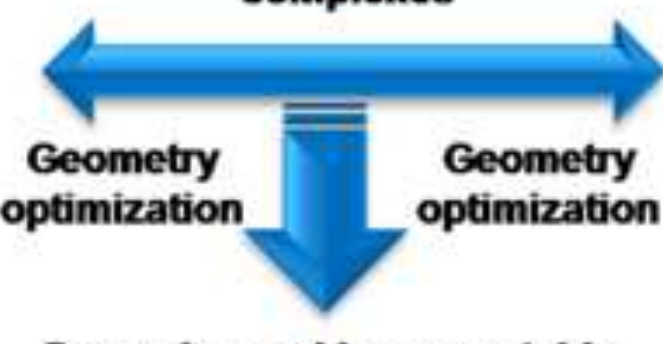

\section{Secondary gel is more stable} to avoid component segregation

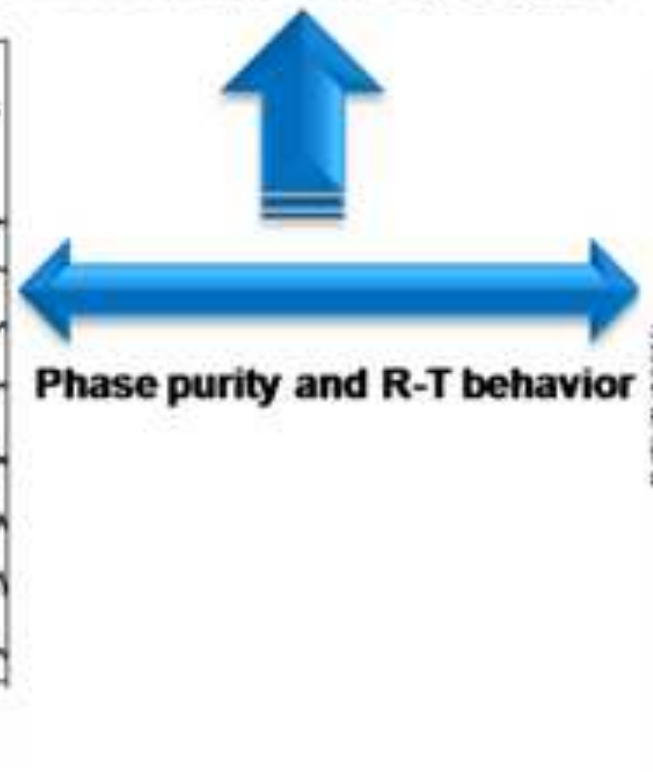

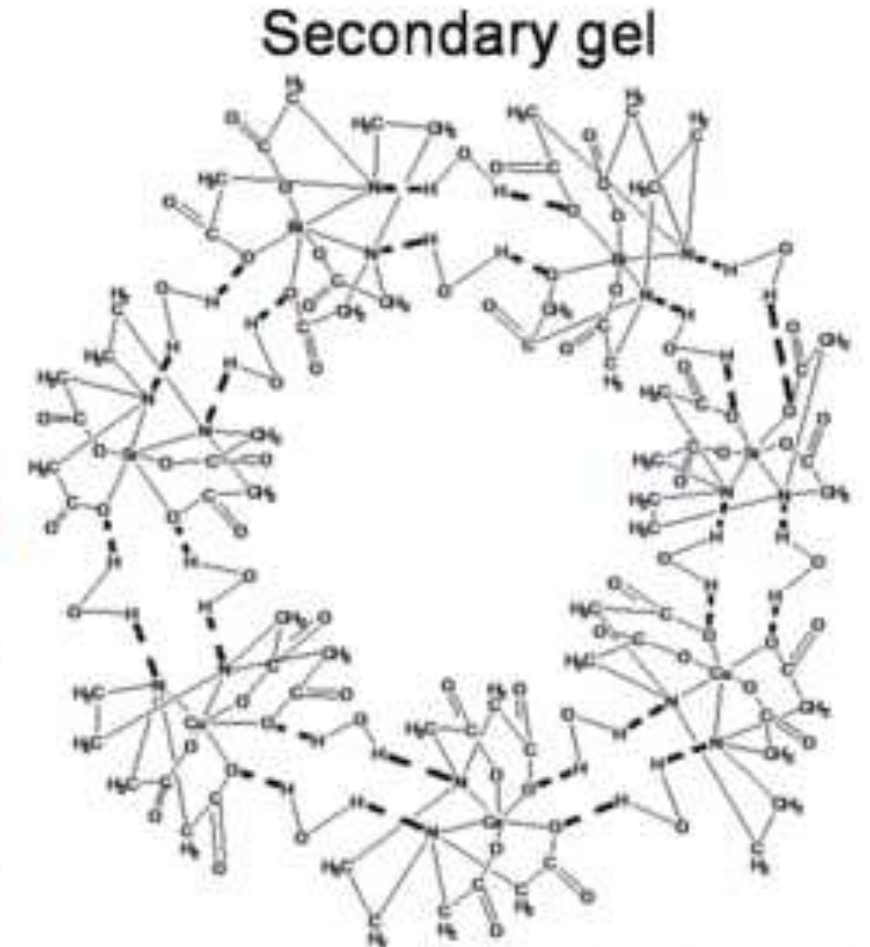

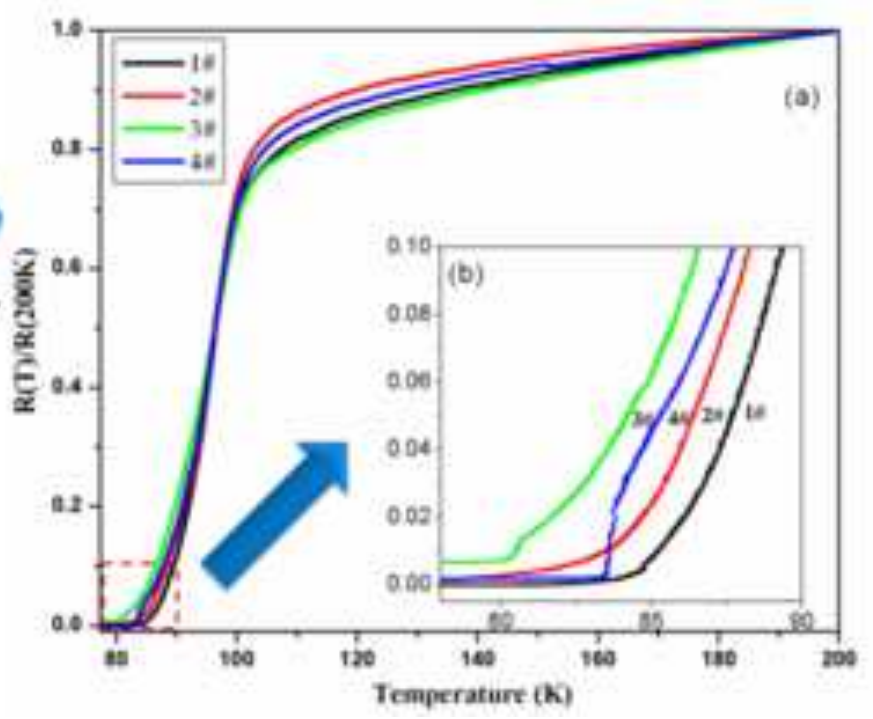

